EPJ manuscript No.

(will be inserted by the editor)

\title{
Static-light mesons on a dynamical anisotropic lattice
}

\author{
Justin Foley $^{1}$, Alan ó Cais ${ }^{2}$, Mike Peardon², Sinéad M. Ryan² \\ 1 Department of Physics, Swansea University, Singleton Park, Swansea SA2 8PP, UK \\ 2 School of Mathematics, Trinity College, Dublin 2, Ireland \\ Received: date / Revised version: date
}

\begin{abstract}
We present results for the spectrum of static-light mesons from $\mathrm{N}_{\mathrm{f}}=2$ lattice QCD. These results were obtained using all-to-all light quark propagators on an anisotropic lattice, yielding an improved signal resolution when compared to more conventional lattice techniques. In particular, we consider the inversion of orbitally-excited multiplets with respect to the 'standard ordering', which has been predicted by some quark models.
\end{abstract}

PACS. PACS-key 11.15.Ha - PACS-key 12.38.Gc

\section{Introduction}

It has long been known that hadrons containing a single charm or bottom quark exhibit approximate heavy quark spin and flavour symmetries and can be described to a reasonable approximation by an idealised system in which the heavy quark is taken to be infinitely massive [1. In this limit the heavy quark symmetries become exact and the system consists of light degrees of freedom bound to a static colour point source. The static approximation can be systematically improved using a power-counting rule in $\Lambda_{\mathrm{QCD}} / \mathrm{m}_{\mathrm{Q}}$, which forms the basis for heavy quark effective theory (HQET). For hadrons containing a bottom quark this approach is particularly suitable. In this case one expects leading-order corrections to be already quite small, at the level of ten percent.

On the lattice, mass-dependent errors which arise from the discretisation of the Dirac operator can limit the accuracy of simulations of heavy-light systems. In principle, one can avoid such errors by using the HQET lagrangian to describe the heavy quark dynamics. An alternative approach is to perform a number of simulations both at unphysically light heavy quark masses using the lattice Dirac operator and in the static limit and to interpolate the results to the physical heavy quark mass guided by the predictions of HQET. Therefore, precise calculations in the static limit are of considerable interest to the lattice community. In addition, little is known experimentally of the excited state spectrum of heavy-light hadrons and accurate simulations of their simpler static-light counterparts will have considerable phenomenological impact. However, traditionally, simulations of static-light systems have been extremely noisy. Ultimately, this stems from the fact that the use of conventional point-to-all propagators for the light quarks restricts interpolating operators for the static-light hadron to a single spatial lattice site.
This problem could be overcome if it were possible to compute all elements of the light quark propagator. This would allow source and sink operators for the static-light correlators to be placed at every spatial lattice site, yielding a dramatic increase in statistics. Indeed, simulations of static-light mesons are the natural testing ground for all-to-all techniques since they require a single light quark propagator per configuration while the static quark propagator is easy to evaluate. It is therefore not surprising that a number of groups have attempted to tackle this particular problem [2:3].

In this contribution, we describe a preliminary study of the excited state spectrum of static-light mesons using the all-to-all propagator technique introduced in Ref. [4. In this approach, the important contribution of the low-lying eigenmodes of the Dirac operator to the fermion propagator is computed exactly. A noisy estimate is then used to correct for the contribution of the remaining eigenmodes. This estimate is obtained by inverting the Dirac operator on a set of $\mathrm{Z}_{4}$ noise sources which have been partitioned in some subset of quarkfield indices. This partitioning or 'dilution' yields a substantial reduction in the variance of the stochastic estimate. This study has been performed on a $3+1$ anisotropic lattice, where the temporal lattice spacing $a_{t}$ is much finer than the spatial lattice spacing $a_{s}$. The fine temporal lattice spacing proves particularly useful when determining a plateau in the effective masses of the higher excited states. The correlators have been computed on $\mathrm{N}_{\mathrm{f}}=2$ background configurations.

\section{Interpolating operators}

Due to heavy quark spin symmetry, states which lie in the same hyperfine multiplet are degenerate and in the continuum it is conventional to label these multiplets by the 


\begin{tabular}{|c|c|c|}
\hline Lattice irrep & Dimension & $J$ \\
\hline $\mathrm{G}_{1}$ & 2 & $\frac{1}{2}, \frac{7}{2} \ldots$ \\
\hline $\mathrm{G}_{2}$ & 2 & $\frac{5}{2}, \frac{7}{2} \ldots$ \\
\hline $\mathrm{H}$ & 4 & $\frac{3}{2}, \frac{5}{2}, \frac{7}{2} .$. \\
\hline
\end{tabular}

Table 1. Irreducible representations of the group of 24 proper spatial lattice rotations with low-lying angular momentum content.

angular momentum and parity of the light degrees of freedom [5]. For example, in the static limit the pseudoscalar and vector correspond to $J_{\ell}{ }^{P_{\ell}}=\frac{1}{2}^{-}$.

At finite lattice spacing, it makes sense to classify states according to the irreducible representations (irreps) of the octahedral point group $\mathrm{O}_{h}$ [6]. This 48 element group is the direct product of the 24 proper spatial rotations allowed by the lattice with the group consisting of the identity and the space inversion operator. The relationship between these representations and continuum quantum numbers is not difficult to determine. Restricting the continuum irreps to the elements of the lattice symmetry group generates representations for $\mathrm{O}_{\mathrm{h}}$ which are in general reducible and identification of the constituent irreps is a straight-forward exercise in group theory. Here, we take advantage of the degeneracies which arise from the heavy quark spin symmetry and construct interpolating operators specifically for the light degrees of freedom. Therefore, our operators transform according to the double-valued or spinorial representations of $\mathrm{O}_{\mathrm{h}}$. There are 6 such representations

$$
\mathrm{G}_{1 \mathrm{u}}, \mathrm{G}_{1 \mathrm{~g}}, \mathrm{G}_{2 \mathrm{u}}, \mathrm{G}_{2 \mathrm{~g}}, \mathrm{H}_{\mathrm{u}}, \mathrm{H}_{\mathrm{g}} .
$$

where the subscripts $u$ and $g$ denote representations which are odd and even under spatial inversion respectively.

Table 1 lists the irreps of the subgroup of proper rotations and the angular momenta of their lower-lying constituent states. From this table we see that states with the same angular momentum but with different $J_{z}$ may be scattered across the lattice irreps. For example, states which transform according to the rows of the 6-dimensional spin $\frac{5}{2}$ representation are divided between the $G_{2}$ and $H$ irreps. In a numerical study we should therefore expect to observe energy levels in these irreps which are degenerate, up to lattice artifacts, corresponding to the $J_{\ell}=\frac{5}{2}$ states. In this study, we were particularly interested in orbital excitations of the static-light meson. There is a single S-wave state with $J_{\ell}^{P_{\ell}}$ quantum numbers $\frac{1}{2}^{-}$, the P-wave doublet is $\frac{1}{2}^{+}, \frac{3}{2}^{+}$and the D-wave states are labelled $\frac{3}{2}^{-}, \frac{5}{2}^{-}$. Of these, only the S-wave and the $\frac{1}{2}^{+} \mathrm{P}$-wave which lie in the $\mathrm{G}_{1 \mathrm{u}}$ and $\mathrm{G}_{1 \mathrm{~g}}$ irreps can be accessed using local operators, while all other irreps require the use of extended interpolating operators. It is worth noting that when one uses all-to-all light quark propagators the construction of extended interpolating fields requires no additional fermion matrix inversions and the extra computational cost incurred is minimal. In order to determine accurately the excited state spectrum it is important to choose operators which couple strongly to the states of interest and we tested a number of operators across the lattice representations. Further details of our choice of interpolating operators will be presented in Ref. [7].

\section{Simulation details}

The lattice Dirac and gauge actions used in this study have previously been described in detail in Refs. 8. 9. Both are specifically formulated for $3+1$ anisotropic lattices. The Wilson-like fermion action is accurate to $\mathcal{O}\left(a_{t}, a_{s}^{3}\right)$ and employs stout-smeared spatial links to reduce radiative corrections. The gauge action incorporates tree-level Symanzik and tadpole improvement and is accurate to $\mathcal{O}\left(a_{t}^{2}, a_{s}^{4}, \alpha_{s} a_{s}^{2}\right)$.

The anisotropic lattice breaks hypercubic symmetry and introduces two new parameters: the bare quark and gauge anisotropies, denoted $\xi_{q}^{0}$ and $\xi_{g}^{0}$, which must be tuned such that the measured anisotropy, $a_{s} / a_{t}$, assumes a consistent value. In dynamical QCD the tuning procedure is quite complicated and is detailed in Ref. [10]. In this study we use bare anisotropy values taken from that paper of $\xi_{q}^{0}=7.52$ and $\xi_{g}^{0}=8.06$ which yields a measured anisotropy of 6 with a three percent error.

We employ the standard Eichten-Hill action 11 for the static quark and the corresponding static quark propagator is a product of unsmeared temporal links.

Measurements have been performed on 249 background configurations on an $8^{3} \times 80$ lattice with a spatial lattice spacing of about $0.2 \mathrm{fm}$. The mass of the sea quarks and the light valence quark was found to be approximately the strange quark mass. For the light quark propagator, we computed the contribution of 100 low-lying eigenvectors of the Dirac operator. Two independent $\mathrm{Z}_{4}$ noise sources which were diluted in time and colour were used in the stochastic estimate.

To extract excited state energies we applied the standard variational analysis to correlation matrices which were obtained by applying 5 or 6 levels of Jacobi smearing to the light quark fields. This was facilitated by the use of all-to-all propagators which meant that no additional inversions were required to smear the quark fields.

\section{Results}

Fig. 11 shows effective masses for the lowest-lying states across a number of the lattice irreps. In this case the lowest effective mass corresponds to the S-wave $\frac{1}{2}^{-}$state and the higher-lying states are orbital excitations. The highestlying states lie in D-wave channels. Clear plateaux are evident in each channel.

In addition, we obtain strong signals for a number states in each of the lattice irreps. Fig. 2 shows the ground state and the first two excited states in the $\mathrm{G}_{1 \mathrm{u}}$ (S-wave) irrep. These excited states shown here are almost certainly radial excitations in the $\mathrm{S}$-wave channel.

Since we are working in the static limit the energies determined by fitting to the effective masses contain an 


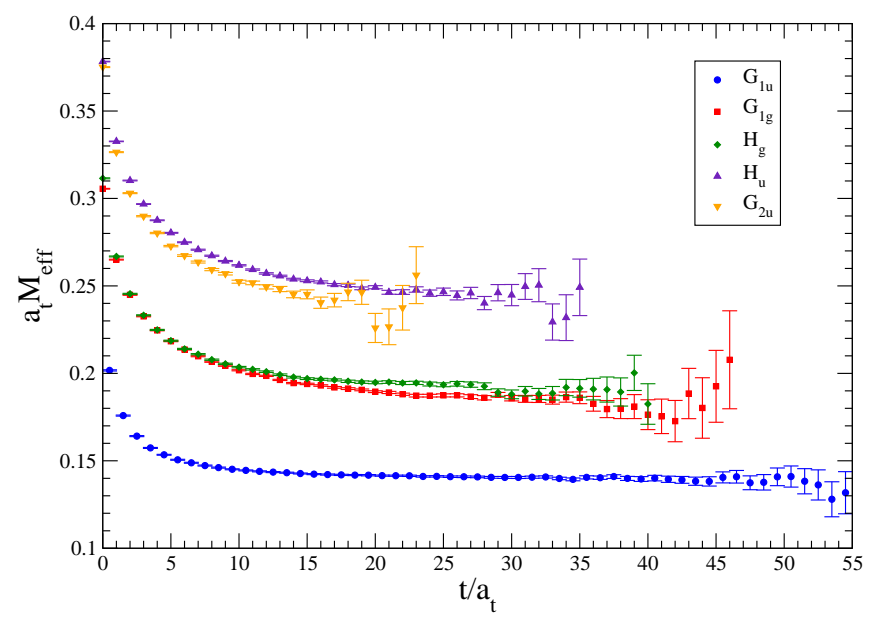

Fig. 1. Effective masses for the lowest-lying states in 5 lattice irreps. These include $\mathrm{P}$-wave and $\mathrm{D}$-wave excitations.

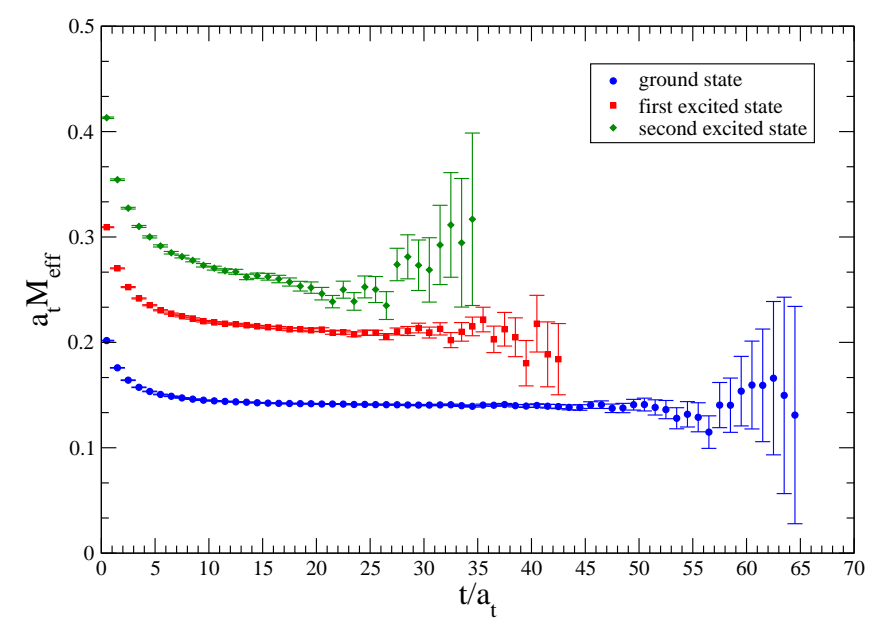

Fig. 2. Effective masses for the lowest-lying states in the $G_{1 u}$ (S-wave) irrep.

unphysical shift. This shift cancels in energy differences, which are therefore physically meaningful. Fig. 3 plots the energy differences between the lowest-lying state in the $\mathrm{G}_{1 \mathrm{u}}$ representation (i.e. the $\mathrm{S}$-wave multiplet) and a number of excited states. We have not presented these preliminary results in physical units because we have performed our simulation on quite a small spatial volume which might distort the energies of the orbitally and radially excited states. The main point here is the precision to which we can compute the spectrum. Nevertheless, it is interesting to note the position of the lowest-lying states in the $\mathrm{H}_{\mathrm{u}}$ and $\mathrm{G}_{2 \mathrm{u}}$ irreps. Naively, one expects the lightest state in the $\mathrm{H}_{\mathrm{u}}$ representation to correspond to the $\frac{3}{2}^{-}$D-wave while the $\frac{5}{2}^{-}$ought to be the lightest state in the $\mathrm{G}_{2 \mathrm{u}}$ irrep. However, there appears to be no significant splitting between these states which may indicate that the $\frac{5}{2}^{-}$multiplet is lighter than the $\frac{3}{2}^{-}$. Such a scenario has been predicted by quark models [12 13 .

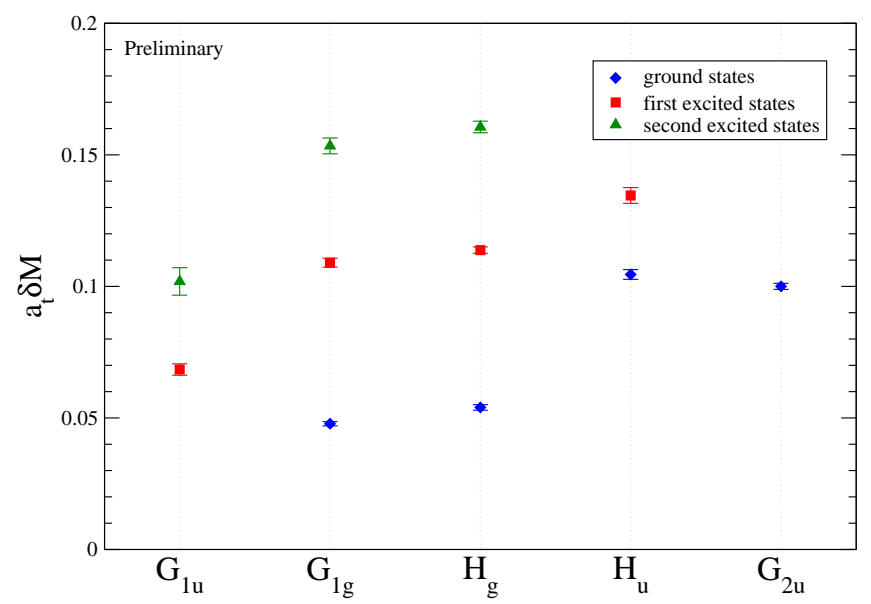

Fig. 3. Mass differences between the ground state S-wave multiplet and higher lying states.

\section{Conclusions and outlook}

We have presented preliminary results for the spectrum of static-light mesons in $\mathrm{N}_{\mathrm{f}}=2 \mathrm{QCD}$. We have been able to compute energy differences between the S-wave multiplet and a number of orbital and radial excitations. The use of all-to-all propagators allows us to minimise the statistical uncertainty in our measurements and facilitates the use of spatially-extended interpolating operators and the variational approach. The results presented here have allowed us to make some tentative remarks about the nature of the spectrum; however, we have not yet made a serious attempt to identify the continuum quantum numbers of the observed states. More recently we have performed similar simulations using larger operator bases on two different spatial volumes. These runs should allow us to assess finite volume effects and identify multi-particle states. We are currently analysing the results of these simulations and we intend to present our findings in the near future [7].

\section{References}

1. N. Isgur and M.B. Wise, Phys. Lett. B232, (1989) 113.

2. A. M. Green et al., Phys. Rev. D69, (2004) 094505.

3. T. Burch and C. Hagen, hep-lat/0607029

4. J. Foley et al., Comput. Phys. Commun. 172, (2005) 145162.

5. N. Isgur and M. B. Wise, Phys. Rev. Lett. 66, (1991) 11301133.

6. S. Basak et al, Phys. Rev. D72, (2005) 094506; Phys. Rev. D72, (2005) 074501.

7. J. Foley et al., in preparation.

8. J. Foley, A. Ó Cais, M. Peardon and S. M. Ryan, Phys. Rev. D72, (2005) 074503.

9. C. Morningstar and M. J. Peardon, Nucl. Phys. Proc. Suppl. 83, (2000) 887.

10. R. Morrin, A. Ó Cais, M. Peardon, S. M. Ryan and J.I. Skullerud, Phys. Rev. D74, (2006) 014505.

11. E. Eichten and B. Hill, Phys. Lett. B240, (1990) 193.

12. H. J. Schnitzer, Phys. Lett. B226 (1989) 171.

13. N. Isgur, Phys. Rev. D57, (1998) 4041-4053. 
